\title{
ASSOCIATION BETWEEN CONSUMPTION OF PHYTOSTEROLS AND MARKERS OF SUBCLINICAL ATHEROSCLEROSIS IN THE ELSA BRASIL POPULATION
}

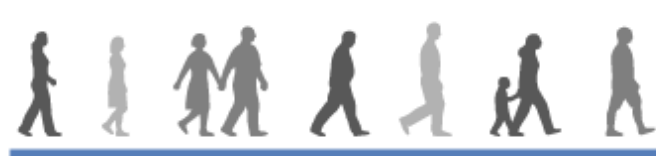 ELSA BRASIL}

T. Pereira1, H.A.R. Fonseca1, F.A.H. Fonseca1, C.M. Martins', I.M. Bensenor'2, P.A. Lotufo², M.C.O. Izar' 1 Universidade Federal de São Paulo, Department of Medicine, São Paulo, Brazil. 2Universidade de São Paulo, Department of Medicine, São Paulo, Brazil

\section{- Background and aims}

The efficacy of plant sterol supplementation, and usual intake of plant sterols, to obtain significant levels of LDL-c reduction, is well documented. However, little is known about the usual consumption of phytosterols on maintenance of serum levels of LDL-C and the presence of subclinical atherosclerosis. The aims of this study were to estimate the usual consumption of phytosterols in the usual diet by the population of ELSA Brazil, and determine its association with markers of subclinical atherosclerosis

\section{- Methods}

We selected 2,560 participants in primary prevention, with available food frequency questionnaires (FFQ), carotid intima-media thickness (cIMT), coronary calcium score (CCS), pulse wave velocity (PWV)

Total energy expenditure (TEE) was $>500 \mathrm{kcal} / \mathrm{d}$ and $\leq 4000 \mathrm{kcal} / \mathrm{d}$.

cIMT and PWV were categorized in quartiles and $<$ or $>$ P75, CCS was categorized as $=$ or $>$ zero

Analysis of the association between phytosterols consumption and subclinical atherosclerosis markers was performed using adjusted and unadjusted logistic and linear models.

- Results

Mean phytosterol consumption was 256.08 ( \pm 197.87$)$, CCS 22.78( \pm 110.53$)$, PWV 9.07( \pm 1.59$)$, and cIMT 0.57( \pm 0.11$)$

Phytosterol consumption, CCS, PWV and CIMT were higher in men $(P<0.001)$. Consumption of phytosterols $>$ P75 was associated with lower levels of total, HDL-c, LDL-c, higher triglycerides $(P<0,01)$, higher TEE, higher consumption of fibres, and PWV $(P<0.01)$.

Univariate logistic and linear regression showed that increased phytosterols consumption levels was associated with increased PWV $(P<0.02$ and $P<0.001$, respectively).

After adjusting for other cardiovascular risk factors, this association disappeared.

Table 1. Characterization of phytosterol consumption and subclinical atherosclerosis markers of participants, by sex

\begin{tabular}{|c|c|c|c|c|}
\hline & Men & Women & Total & P-valor \\
\hline $\mathrm{N}(\%)$ & 1064 & 1496 & 2560 & \\
\hline $\begin{array}{l}\text { Phytosterol } \\
\text { (mg/day) }\end{array}$ & $323.49( \pm 228.43)$ & $208.13( \pm 156.38)$ & $256.08( \pm 197.87)$ & $<0.001$ \\
\hline PWV (m/s) & $9.64( \pm 1.66)$ & $8.66( \pm 1.41)$ & $9.07( \pm 1.59)$ & $<0.001$ \\
\hline $\operatorname{ccs}(\mathrm{UA})$ & $38,65( \pm 141,64)$ & $11,49( \pm 79,63)$ & $22,78( \pm 110,53)$ & $<0,001$ \\
\hline cIMT (mm) & $0.57( \pm 0.11)$ & $0.58( \pm 0.11)$ & $0,57( \pm 0,11)$ & $<0,001$ \\
\hline
\end{tabular}

PWW. Pulse Wave Velocity; CCS, Coronary Calcium Score; c1MTT, Intima Medium Thickness

\section{- Conclusions}

Usual intake of phytosterols from the diet was associated with lower concentrations of total and LDL-cholesterol, with no association with subclinical atherosclerosis markers. This is the largest study evaluating plant sterol consumption in the usual diet and markers of subclinical atherosclerosis, opening perspectives for the clinical importance of natural sources of phytosterols in vascular health.
Table 2. Characteristics of laboratory parameters by percentiles of phytosterol consumption

\begin{tabular}{|c|c|c|c|c|}
\hline $\mathrm{N}(\%)$ & $\begin{array}{c}<\mathrm{p} 75 \\
(<390.85) \\
2010\end{array}$ & $\begin{array}{c}2 p 75 \\
(\geq 390.85) \\
550\end{array}$ & $\begin{array}{l}\text { Total } \\
2560\end{array}$ & P-valo \\
\hline Total (mg/dl) & $216.06( \pm 41.47)$ & $210.73( \pm 39.86)$ & $216.62( \pm 43.85)$ & 0.005 \\
\hline $\begin{array}{l}\text { LDL-c } \\
\text { (mg/dl) }\end{array}$ & $133.21( \pm 33.65)$ & $129.9( \pm 33.97)$ & $132.7( \pm 36.94)$ & 0.036 \\
\hline $\begin{array}{l}\text { HDL-c } \\
\text { (mg/dl) }\end{array}$ & $58.2( \pm 14.58)$ & $53.89( \pm 13.92)$ & $58.43( \pm 13.78)$ & $<0.001$ \\
\hline$\frac{T \text { Triglycerides }}{(\mathrm{m} / \mathrm{dl})}$ & $124.37( \pm 85.20)$ & $139.45( \pm 106.54)$ & $134.33(95.88)$ & 0.001 \\
\hline
\end{tabular}

Table 3. Nutritional characteristics by percentiles of phytosterol consumption

\begin{tabular}{|c|c|c|c|c|}
\hline N (\%) & $\begin{array}{c}p 75 \\
(<390.85) \\
2010\end{array}$ & $\begin{array}{c}p 75 \\
(\geq 390.85) \\
550\end{array}$ & $\begin{array}{l}\text { Total } \\
2560\end{array}$ & P-valor \\
\hline $\begin{array}{l}\text { TEE } \\
\text { (kcaldday) }\end{array}$ & $2395.90( \pm 679.48)$ & $2936.23( \pm 573.29)$ & $2431.18( \pm 689.97)$ & $<0.001$ \\
\hline $\begin{array}{l}\frac{\text { Soluble }}{\text { Fibers }} \\
\text { (g/day) }\end{array}$ & $7.65( \pm 3.43)$ & $8.55( \pm 3.86)$ & $7.86( \pm 3.39)$ & $<0.001$ \\
\hline $\begin{array}{l}\text { Insoluble } \\
\text { Fibers } \\
\text { (g/day) }\end{array}$ & $20.72( \pm 8.07)$ & $34.49( \pm 9.31)$ & $23.17( \pm 9.67)$ & $<0.001$ \\
\hline
\end{tabular}

TEE, total energy expenditure

Table 4. Clinical characteristics among the percentiles of phytosterol consumption

\begin{tabular}{|c|c|c|c|c|}
\hline $\mathrm{N}(\%)$ & $\begin{array}{c}<p 75 \\
(<390.85) \\
2010\end{array}$ & $\begin{array}{c}2 \mathrm{p} 75 \\
(\geq 390.85) \\
550\end{array}$ & $\begin{array}{l}\text { Total } \\
2560\end{array}$ & P-valo \\
\hline $\operatorname{ccs}(U A)$ & $22.70( \pm 115.28)$ & $23.01( \pm 94.95)$ & $22.78( \pm 110.53)$ & 0.396 \\
\hline$\underline{\text { CIMT }(m m)}$ & $0.57( \pm 0.11)$ & $0.57( \pm 0.11)$ & $0.58( \pm 0.11)$ & 0.906 \\
\hline $\mathrm{PWV}(\mathrm{m} / \mathrm{s})$ & $9.00( \pm 1.59)$ & $9.27( \pm 1.59)$ & $9.13( \pm 1.61)$ & $<0.001$ \\
\hline
\end{tabular}

Table 5. Univariate logistic regression analysis, with measurement of PWV $\geq \mathrm{p} 75$ as dependent variable

\begin{tabular}{|c|c|c|}
\hline Variables & OR (95\% IC) & p-valor \\
\hline \multicolumn{3}{|c|}{$\begin{array}{l}\text { Percentile of daily consumption of } \\
\text { phytosterol ( } \mathrm{mg} / \text { day) }\end{array}$} \\
\hline$>$ >p75 (reference) & 100 & \\
\hline$>p 50$ - sp75 & $0.73(0.56-0.95)$ & 0.02 \\
\hline$>p 25-\leq p 50$ & $0.69(0.54-0.88)$ & 0.004 \\
\hline$\leq \mathrm{p} 25$ & $0.69(0.54-0.88)$ & 0.003 \\
\hline
\end{tabular}

PW, pulse wave velocity

Table 6. Multivariate logistic regression analysis with PWV $\geq p 75$ as dependent variable

\begin{tabular}{lcr}
\hline Variables & OR (95\% IC) & p-valor \\
\hline $\begin{array}{l}\text { Percentile of daily consumption of } \\
\text { phytosterol (mg/day) }\end{array}$ & \multicolumn{2}{c}{} \\
\hline$>$ p75 (reference) & 1.00 & \\
\hline$>p 50-\leq p 75$ & $0.87(0.81-1.24)$ & 0.45 \\
\hline$>p 25-\leq p 50$ & $0.85(0.58-1.26)$ & 0.44 \\
\hline$\leq p 25$ & $0.92(0.59-1.43)$ & 0.72 \\
\hline
\end{tabular}

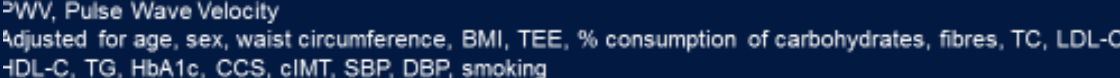

Table 7. Simple linear regression analysis with PWV as dependent variable

\begin{tabular}{lccc}
\hline \hline Variables & Beta & 95\%lC & P valor \\
\hline Phytosterol consumption (mg/day) & 0.08 & $0.00-0.001$ & $<0.001$ \\
\hline
\end{tabular}

